Historic, Archive Document

Do not assume content reflects current scientific knowledge, policies, or practices. 



\title{
Spring 1908
}

A LIST OF CHOICE YOUNG

\section{Ornamental Trees, Shrubs and Vines}

\author{
FOR PLANTING OUT IN NURSERY ROWS
}

To our many customers who regularly buy from us, this list needs no particular recommendation. They will send in their orders at once, that the stock they require may be reserved for them. They know from past experience that to secure their full supply they must order promptly.

To those who have never bought this stock from us we would briefly state that we are probably the largest growers of this small stock in the United States, and our sales each year amount to over a million plants-the stock going into almost every nursery in this country and Canada. We grow an exceptionally fine grade of plants which will give results, and it is absolutely true to name.

Don't delay your order until you are ready to plant. By that time the best of it will be sold. Send in your order now and have the stock reserved for you. It is all in cellars and shipment can be made at any time after the first of February.

\section{FIVE PLANTS OF ANY ONE VARIETY SOLD AT THE RATE QUOTED PER TEN.}

TWENTY-FIVE AT THE RATE QUOTED PER HUNDRED.

TWO HUNDRED AND FIFTY AT RATE QUOTED PER THOUSAND.

\section{PACKING CHARGED EXTRA, AT COST. IF CASH ACCOMPANIES ORDER, PACKING WILL BE FREE.}

\section{THOMAS MEEHAN \& SONS, Inc.} DRESHER'TOWN, MONTG. CO., PA.

Acer campestre, English Cork Bark Maple.......2 year dasycarpum, Silver Maple ............... Io to I5 in.

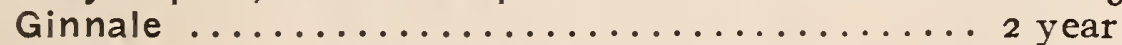
Negundc, Ash-leaved Maple, Box Elder........ 22 to I 8 in. platanoides, Norway Maple............... 6 to 20 in. pseudo-platanus, Sycamore Maple.......... 2 year saccharinum, Sugar Maple .............. 6 to I2 in. saccharinum ..................... 2 to 18 in. saccharinum .................... I to $2 \mathrm{ft}$. striatum (Pennsylvanicum) Striped Maple....2 2 year

Althaea alba plena, double white............ 2 year

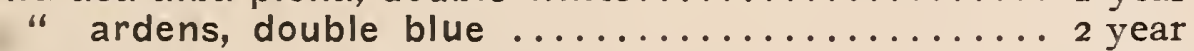
bicolor, double pink.................... 2 year Boule de Feu, double red............... 2 year Duc de Brabant, double red................2 year folius variegata, double variegated leaved....2 2 year Jeanne de Arc, double pure white.......... 2 year Lady Stanley, double pink ............... 2 year totus albus, single pure white........... 2 year

Per 10 Per 100 Per 1000 \$O $35 \$$ I $75 \$$ I5 00 $25 \quad 65 \quad 550$ 35 I 75 I5 OO $25 \quad 75 \quad 600$ 25 I $50 \quad$ I 200 35 I 75 I4 00 25 I 25 I0 00 25 I 50 I3 00 35 I 75 I5 00 $\begin{array}{lllll}50 & 2 & 25 & 18 & 00\end{array}$ 


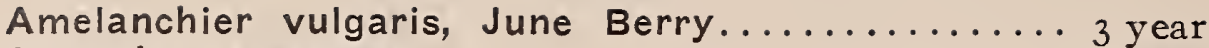

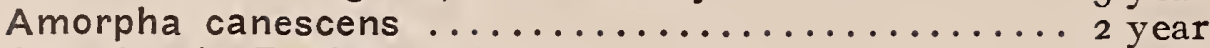

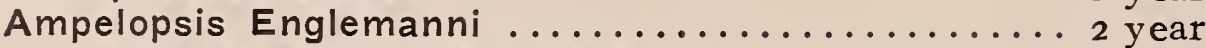

"Virginica, Virginia Creeper ............ 2 year

Aronia floribunda, Choke Berry ............. 2 year

Berberis Thunbergii, Japanese Barberry..........6 to Io in.

" purpurea, Purple Barberry.............. 2 year

" vulgaris, Green Barberry.............. 2 year

Betula alba, European White Birch.............. I8 to 24 in.

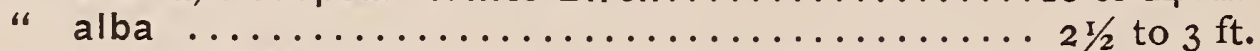

" lenta, Sweet Birch ................ 2 year

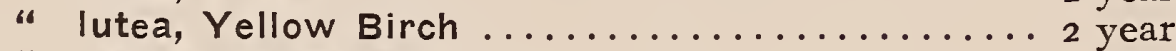

" papyracea, Paper Birch .............. 2 year

" populifolia, Poplar Birch ................ 2 year

Bignonia grandiflora, Orange flowered Trumpet Vine 2 year

Calycanthus floridus, sweet shrub.............. 2 to I8 in.

Caragana arborescens, Siberian Pea............ 2 year

Carpinus Americana, American Hornbeam.......... I2 to I8 in.

Carya alba, Shellbark.................... I year

"olivaeformis, Pecan Nut................6 to Io in.

Castanea Americana, Sweet Chestnut...........6 to I2 in.

" vesca, Spanish Chestnut................ I year

Catalpa speciosa, Western Catalpa................ I2 to I8 in.

Ceanothus Americanus, New Jersey Tea......... 2 year

Celastris scandens, Staff Vine ................. I year

Cephalanthus occidentalis, Dwarf Button Bush....2 2 year

Cercis canadensis, American Judas, Red Bud....... I2 to I8 in.

Chionanthus Virginica, White Fringe.......... 3 to 4 in.

"Virginica ...................... 8 to I2 in.

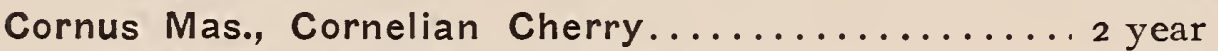

"Siberica, Red Stemmed Dogwood .......... 2 year

Corylus Americana, American Hazel Nut .......... I year

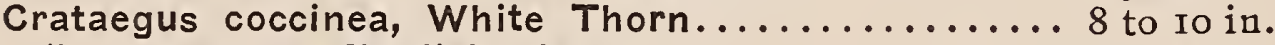

"oxycantha, English Hawthorn............. I to $2 \mathrm{ft}$.

Cydonia Japonica, Red Japanese Quince ......... 2 year

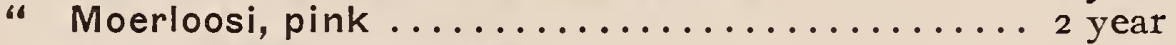

" umbilicata, bright rose ............... 2 year

Deutzia candidissima plena, double white........2 year

" crenata rosea plena, double pink.......... 2 year

" gracilis, Dwarf, white .............. 3 year

"Lemoinei, Dwarf single wnite............ 2 year

“ Pride of Rochester, double pink.......... 2 year

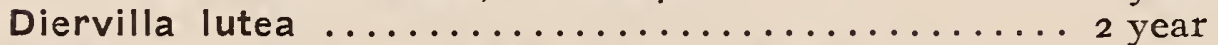

Eleagnus longipes, Large fruited Silver Thorn......2 year

Euonymus Europaeus, European Burning Bush.....2 year

Fagus ferruginea, American Beech............. I2 to I5 in.

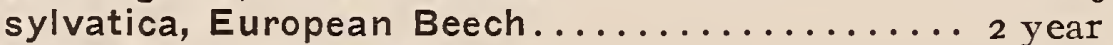

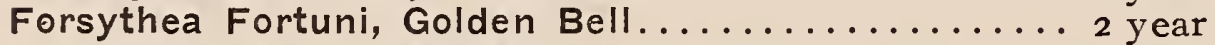
suspensa, Golden Bell ................... 2 year

Fraxinus Americana, American White Ash......... I2 to I8 in.

Halesia tetraptera, Silver Bell............. 2 year

Hamamelis Virginica, Wych-hazel ............... I2 to I5 in.

Hippophaea rhamnoides, Sea Buckthorn.........2 year

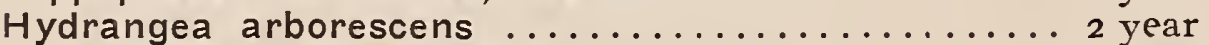
paniculata grandiflora ..................... I year strong

Humulus lupulus, Hop-vine ................ 2 year

Juglans nigra, Black Walnut ............... I to $2 \mathrm{ft}$.

regia, English Walnut, Madeira Nut ......... 2 year

Kerria Japonica fl. pl. double (Chochorus)........2 year

" Japonica, single ................. 2 year

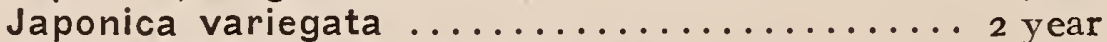

Koelreuteria paniculata, Varnish Tree.......... 3 year

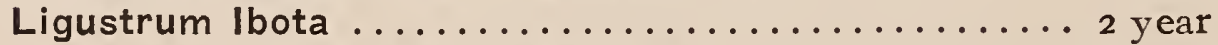

"ovalifolium, California Privet ............. I2 to I5 in.

" vulgaris, Common Privet ................ I year

Liriodendron tulipfera, Tulip Tree............. 2 to I8 in.
Per 10 Per 100 Per 1000

$\$ 050 \$ 250 \$ 2200$

35200 I6 00

$\begin{array}{lllll}50 & 2 & 25 & 20 & 00\end{array}$

25 I 50 I 2 OO

$\begin{array}{lllll}50 & 3 & 50 & 29 & 00\end{array}$

35 I 75 I5

35 I 75 I5 00

35 I 75 I5

25 I 25 I I 00

25 I 50 I3 00

$\begin{array}{lllll}50 & 2 & 25 & \text { I8 } & 00\end{array}$

$\begin{array}{lllll}50 & 2 & 25 & \text { I9 } & 00\end{array}$

$\begin{array}{lllll}50 & 2 & 25 & \text { I8 } & \text { OO }\end{array}$

$\begin{array}{lllll}50 & 2 & 25 & \text { I8 } & \text { OO }\end{array}$

$\begin{array}{lllll}75 & 5 & 00 & 42 & 00\end{array}$

$35200 \quad 1600$

35 I 75 I5

25 I 50 I3 00

$\begin{array}{lllll}50 & 2 & 25 & 20 & 00\end{array}$

$35 \quad 250 \quad 2500$

35 I 75 I4

35 I 75 I4 OO

$25 \quad 75 \quad 600$

35200 I6 OO

$\begin{array}{lllll}60 & 3 & 75 & 32 & 00\end{array}$

$\begin{array}{lllll}50 & 2 & 25 & 20 & 00\end{array}$

25 I 25 IO 00

$\begin{array}{lllll}35 & \text { I } & 75 & \text { I } 5 & 00\end{array}$

$\begin{array}{lllll}50 & 3 & 25 & 30 & 00\end{array}$

352 OO I6 OO

$\begin{array}{lllll}35 & \text { I } & 75 & \text { I } 5 & \text { OO }\end{array}$

$\begin{array}{lllll}50 & 2 & 50 & 22 & 00\end{array}$

$\begin{array}{lllll}50 & 2 & 25 & \text { I } 8 & 00\end{array}$

35 I 75 I4 00

35 I 75 I 500

$\begin{array}{lllll}50 & 2 & 75 & 25 & 00\end{array}$

$50250 \quad 2200$

25 I 50 I 3 OO

25 I 50 I3

25 I 50 I 300

25 I 50 I 300

25 I 50 I3 00

$\begin{array}{lllll}50 & 2 & 25 & 20 & 00\end{array}$

$\begin{array}{lllll}50 & 2 & 25 & \text { IS } & \text { OO }\end{array}$

35200 I6 OO

35 I $75 \quad$ I 6 OO

$\begin{array}{lllll}50 & 2 & 25 & \text { I8 } & \text { OO }\end{array}$

$\begin{array}{lllll}50 & 2 & 75 & 25 & 00\end{array}$

$\begin{array}{lllll}50 & 3 & 00 & 27 & 00\end{array}$

25 I 50 I 3 OO

$50 \quad 250 \quad 2200$

352 OO I6 OO

$\begin{array}{lllll}35 & 2 & 00 & 15 & 00\end{array}$

$\begin{array}{lllll}50 & 2 & 75 & 25 & \text { no }\end{array}$

$\begin{array}{lllll}50 & 2 & 25 & 20 & 00\end{array}$

$\begin{array}{lllll}35 & 2 & \text { OO I7 } & \text { OO }\end{array}$

$\begin{array}{lllll}35 & 2 & 00 & \text { I } 5 & 00\end{array}$

$\begin{array}{lllll}35 & 2 & 00 & I 7 & 00\end{array}$

$\begin{array}{lllll}50 & 2 & 25 & \text { I9 } & 00\end{array}$

50 2252000

$\begin{array}{lllll}50 & 2 & 25 & \text { I9 } & 00\end{array}$

$\begin{array}{lllll}35 & 2 & \text { OO } & \text { I7 } & 00\end{array}$

$\begin{array}{lllll}35 & \text { I } & 75 & \text { I } 5 & \text { OO }\end{array}$

35 I $75 \quad 1500$

$\begin{array}{lllll}50 & 3 & 25 & 30 & 00\end{array}$

25 I 25 IO 00 
Lonicera glauca ....................................... year grandiflora rosea, Pink Bush Honeysuckle .... 2 year

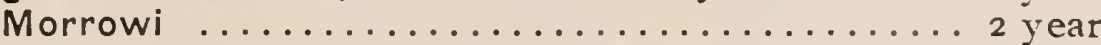
punicea, Scarlet Trumpet Honeysuckle ...... 2 year

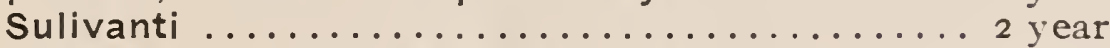
tatarica alba, White Bush Honeysuckle ....... 2 year

Magnolia acuminata, Cucumber Tree........... I year

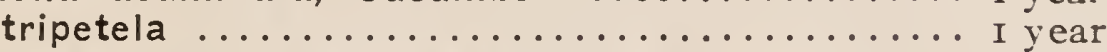

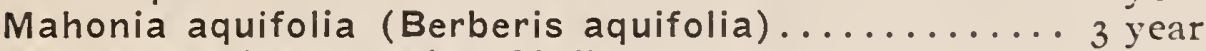
Morus tatarica, Russian Mulberry.............. 2 to IS in.

Philadelphus aurea, Golden Mock Orange ......... 2 year " coronarius, Sweet flowered Mock Orange..... 2 year "grandiflorus, Large flowered Mock Orange.... 2 year

Platanus orientalis, European Sycamore or Plane... 2 year

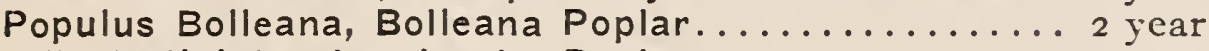
"fastigiata, Lombardy Poplar.............. 2 year

Pyrus floribunda, Choke Berry-see Aronia.........

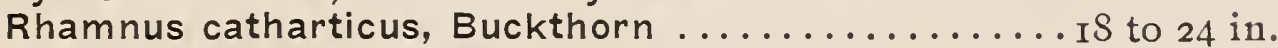

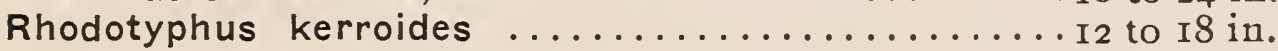
" kerroides ......................... Is to 24 in.

Rhus cotinus, Mist or Smoke Bush ............ 2 year

" glabra laciniata, Cut-leaved Sumach........2 year

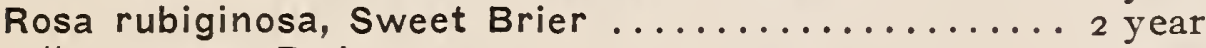

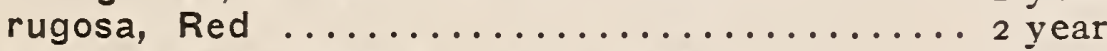

Rubus Canadensis rosea, Flowering Raspberry...... 2 year

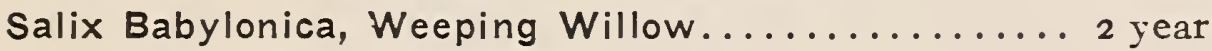

"Britzensis, Red barked Willow........... 2 year " regalis, Royal or White-leaved Willow........ 2 year " rosmarinifolia, Rosmary Willow............ 2 year " vitellina aurea, Golden barked Willow........2 year

Sambucus aurea, Golden-leaved Elder ........... 2 year

" racemosus, Red-berried Elder............... I year

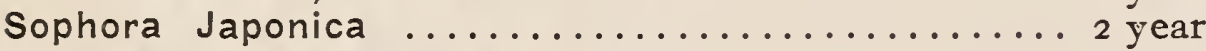

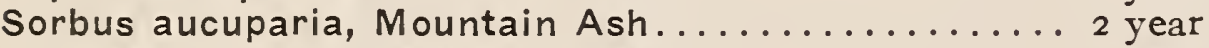

Spiraea Anthony Waterer, Dwarf Pink........... 2 year

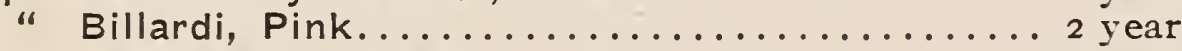

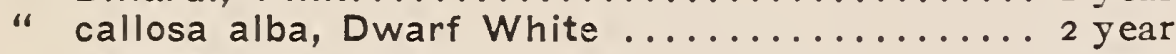

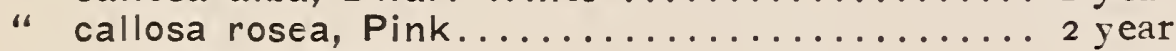

" Douglassi rosea, Pink ................. 2 year

" opulifolia, Nine Bark ................. 2 year

"opulifolia aurea, Golden leaved............ 2 year

" prunifolia, Bridal Wreath .............. 2 year

"Reevesiana, Single flowered, White.......... 2 year

"Reevesiana fl. pl., Double flowered, White..... 2 year

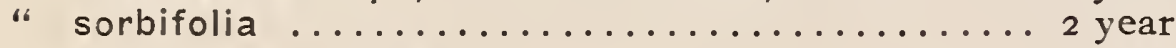

"Thunbergii, white ................. 2 year

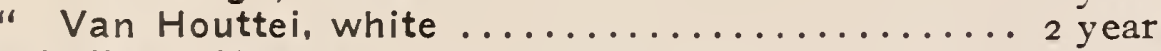

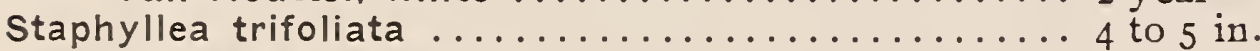

Styrax obassia ..................... 5 to 6 in.

Symphoricarpus racemosus, White Snowberry.....2 2 year

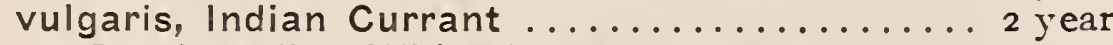

Syringa Persica alba, White Persian Lilac ....... 2 year

"Rothomagensis, Red Persian Lilac..........2 year

"vulgaris, Common Purple Lilac............ 2 year

"vulgaris alba, Common white Lilac.......... 3 year

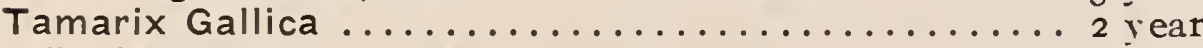

"Japonica plumosa ................... 2 year

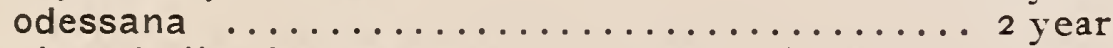

Tilia plataphylla, Large-leaved European Linden... . 2 year

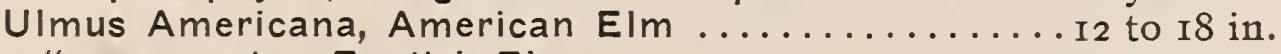

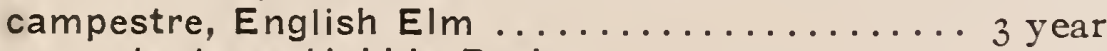

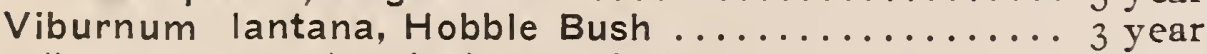
"oxycoccos (opulus), Highbush Cranberry .....2 year

" opulus fl. pl., Common Snowball ...........2 year

" plicatum, Japanese Snowball .............. I year

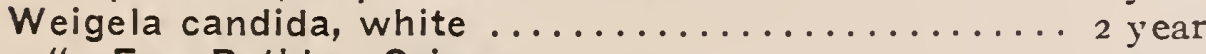

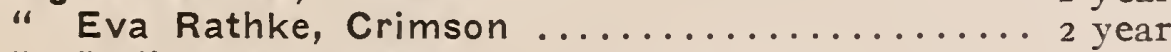

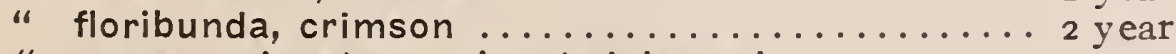

" nana variegata, variegated leaved.......... 2 year

" rosea, pink
Per 10 Per 100 Per 1000

$\$ 075 \$ 5$ oo $\$ 40$ oo

$\begin{array}{lllll}50 & 2 & 25 & 18 & 00\end{array}$

$\begin{array}{lllll}50 & 3 & 75 & 36 & 00\end{array}$

$\begin{array}{lllll}65 & 4 & 00 & 37 & 00\end{array}$

$\begin{array}{lllll}50 & 3 & 25 & 3 \mathrm{I} & 00\end{array}$

$\begin{array}{lllll}50 & 2 & 25 & \text { I9 } & \text { OO }\end{array}$

$\begin{array}{lllll}50 & 3 & 75 & 34 & 00\end{array}$

$\begin{array}{lllll}50 & 2 & 25 & 20 & 00\end{array}$

$\begin{array}{lllll}35 & 2 & \text { OO } & \text { I5 } & 00\end{array}$

$\begin{array}{llll}25 & 65 & 5 & 50\end{array}$

$\begin{array}{lllll}50 & 3 & 25 & 3 \text { I } & 00\end{array}$

$\begin{array}{lllll}50 & 2 & 50 & 23 & 00\end{array}$

$\begin{array}{lllll}50 & 2 & 25 & \text { I9 } & 00\end{array}$

$\begin{array}{lllll}50 & 2 & 25 & 20 & 00\end{array}$

$\begin{array}{lllll}50 & 3 & 25 & 30 & 00\end{array}$

$35200 \quad 1600$

35 I 75 I 5

25 I 25 IO 00

$\begin{array}{lllll}35 & \text { I } & 75 & \text { I } 5 & \text { OO }\end{array}$

$\begin{array}{lllll}50 & 2 & 25 & 20 & 00\end{array}$

$\begin{array}{lllll}65 & 5 & 00 & 45 & 00\end{array}$

$\begin{array}{lllll}35 & 2 & 00 & 17 & 00\end{array}$

$\begin{array}{lllll}50 & 2 & 25 & 20 & 00\end{array}$

$50350 \quad 29 \mathrm{co}$

$\begin{array}{lllll}50 & 2 & 25 & 20 & 00\end{array}$

$35200 \quad 16$ oo

$\begin{array}{lllll}50 & 2 & 25 & 20 & 00\end{array}$

$\begin{array}{lllll}50 & 2 & 25 & \text { I9 } & 00\end{array}$

$\begin{array}{lllll}35 & 2 & \text { OO I7 } & \text { OO }\end{array}$

$\begin{array}{lllll}50 & 2 & 75 & 24 & 00\end{array}$

$\begin{array}{lllll}50 & 2 & 25 & 20 & 00\end{array}$

$\begin{array}{lllll}35 & 2 & \mathrm{OO} & \mathrm{I} 7 & \mathrm{OO}\end{array}$

$\begin{array}{lllll}35 & \text { I } & 75 & \text { I } 5 & \mathrm{OO}\end{array}$

$\begin{array}{lllll}50 & 2 & 25 & 20 & 00\end{array}$

$\begin{array}{lllll}50 & 2 & 25 & \text { I8 } & 00\end{array}$

$\begin{array}{llllll}35 & 2 & 00 & \text { I } 7 & 00\end{array}$

$\begin{array}{lllll}35 & 2 & \mathrm{OO} & \mathrm{I} 7 & \mathrm{OO}\end{array}$

352 OO I7 00

35200 I6 00

$\begin{array}{lllll}50 & 2 & 75 & 24 & 00\end{array}$

$\begin{array}{lllll}50 & 3 & 75 & 35 & 00\end{array}$

$\begin{array}{lllll}50 & 2 & 25 & 18 & 00\end{array}$

$\begin{array}{lllll}50 & 2 & 25 & \text { IS } & 00\end{array}$

$50 \quad 225 \quad 2000$

$50 \quad 2 \quad 25 \quad 2000$

$\begin{array}{lllll}50 & 2 & 25 & 20 & 00\end{array}$

25 I 50 I2 00

35 I 75 I5

$\begin{array}{lllll}35 & 2 & \mathrm{OO} & \mathrm{I} 7 & \mathrm{OO}\end{array}$

$\begin{array}{lllll}35 & 2 & \text { OO } & \text { I } 7 & 00\end{array}$

$\begin{array}{lllll}50 & 3 & 75 & 35 & 00\end{array}$

$\begin{array}{lllll}50 & 3 & 25 & 30 & \mathrm{co}\end{array}$

$\begin{array}{lllll}35 & \text { I } & 75 & \text { I5 } & \text { OO }\end{array}$

$\begin{array}{lllll}50 & 2 & 75 & 25 & 00\end{array}$

$\begin{array}{lllll}35 & 2 & 00 & 17 & 00\end{array}$

50 $250 \quad 2300$

$\begin{array}{llllll}50 & 2 & 50 & 21 & 00\end{array}$

$\begin{array}{lllll}35 & \text { I } & 75 & \text { I } 5 & 00\end{array}$

$25 \quad 65 \quad 550$

$35 \simeq 75$ I5 00

$\begin{array}{lllll}35 & 2 & \text { OO } & \text { I } 7 & \text { OO }\end{array}$

$\begin{array}{lllll}50 & 2 & 50 & 22 & 00\end{array}$

$\begin{array}{lllll}50 & 2 & 75 & 25 & 00\end{array}$

$\begin{array}{lllll}50 & 3 & 25 & 30 & 00\end{array}$

$50350 \quad 3200$

$\begin{array}{lllll}65 & 5 & 00 & 46 & 00\end{array}$

$\begin{array}{llll}50 & 2 & 25 & \text { I8 }\end{array}$

$50 \quad 275 \quad 2400$

$\begin{array}{lllll}50 & 2 & 25 & 31 & 0 O\end{array}$ 


\title{
THE BEST RAFFIA \\ Red Star Brand
}

NEW PRICES effective January 1st, 1908, and continue until further notice.

There is probably nothing that will cause a nurseryman more annoyance, loss of time, and loss of money than a poor quality of Raffia, yet how few nurserymen consider this when purchasing their supply. A first quality Raffia costs little more, if any, than an inferior grade, but in the long run many dollars can be saved. Promiscuous brands have no standard, they may be low in price, but you are buying a "pig in a bag." You have no means of knowing the quality until you get it and if it is not good you have no redress because no standard has been fixed. Red Star Brand Raffia is not sold in that way. It has a standard quality in strength, color and good length of strands and back of that stands our guarantee that it is as represented or money refunded. No other dealer in Raffia will give you this guarantee. They cannot because they do not know the quality of their promiscuous brands. It took us years to work up a source of supply for a standard, uniform quality, and now that we have it we can guarantee it and our guarantee holds good.

There is more Red Star Brand Raffia sold to nurserymen in the United States than all the promiscuous brands put together. Through an advantageous contract covering the next few months we are able to quote the following prices. To secure these prices you should place your order immediately and we will hold the goods for shipment any time between now and the first of June. TERMS, net cash thirty days from date of shipment.

I 1 b. at I8c. per $1 \mathrm{~b}$. 25 lbs. at I Ic. per $1 \mathrm{~b}$.

$501 \mathrm{bs}$. at $10 \mathrm{I} / 2 \mathrm{c}$. per $1 \mathrm{~b}$. Ioo lbs. at 9c. per $1 \mathrm{~b}$.
2 bales at $8 \frac{1}{4}$ c. per $1 b$.

5 bales at $8 \mathrm{c}$. per $1 \mathrm{~b}$. I bale (about 225 lbs.) at $8 \mathrm{r} / 2 \mathrm{c}$. per $1 \mathrm{~b}$.

Special Prices on application for lots of ten and twenty bales.

\section{Hydrangea Paniculata Grandiflora}

STRONG ONE YEAR PLANTS FOR SETTING IN NURSERY ROWS.

Ourstrong one year plants are equal in size and vigor to the two year imported cuttings, and the percentage of loss in transplanting is far less. We hare only a limited quantity to offer this year. Order at once. Shipment can be made at any time.

Price 50c. per Io; $\$ 2.25$ per IOo; $\$ 20.00$ per I000.

\section{FRUIT SEEDS}

\begin{abstract}
APPLE, FRENCH CRAB - New crop is an entire failure. We have none to offer. We have a limited quantity of good, one year old seed. Most nurserymen are buying this. Sample on application. 40c. per lb., $\$ 1200$ per bu., 10 bus. or over at $\$ 11.50$ per bu. CHERRY, MAHALEB.-New crop stratifled in sand. $40 \mathrm{c}$. per lb., $\$ 17.00$ per bu , 3 bus. at $\$ 16.00$ per bu., 5 bus at $\$ 15.00$ per bu.

PEAR, FRENCH.-Crop a complete failure-none to offer.

PEAR, KIEFER. -New crop. Fine quality. $\$ 2.25$ per $1 \mathrm{~b}$., $251 \mathrm{lbs}$. at $\$ 2.00$ per $1 \mathrm{~b}, 50 \mathrm{lbs}$. at $\$ 1.75$ per $1 \mathrm{~b}$.

PLUM, MYROBOLAN.-New crop stratified in sand. 40c. per 1 b., $\$ 11.00$ per bu., 3 bus. at $\$ 10.00$ per bu., 5 bus. at $\$ 9.25$ per bu.

QUINCE.-New crop. $\$ 1.50$ per $1 \mathrm{~b},, 5 \mathrm{lbs}$. at $\$ 1.25$ per $1 \mathrm{~b},, 10$ lbs. at $\$ 1.00$ per $1 \mathrm{~b}$.
\end{abstract}

Postage.-When seeds are to be sent by mail add Ioc. per $1 b$. for postage.

Good Knives For budding, our "Empire" is the best. All ivory tapered handle. Patented after very popular. 35c. each, 6 for $\$ 2.00$. "C" a substantial knife. Stationary blade. 25c. each, 6 for $\$ 1.30$. Two good Pruning Knives- "A" a lightweight knife for general use. Ebon y handle. 75c. each, 6 for $\$ 3.75$. "B," the nurserymen's choice. Strong blade. Round handle. steel cap on end. Far superior to "A," $85 \mathrm{c}$. each, 6 for $\$ 4.75$. Prices include cost of postage. PRUNING SHFARS- "E," 75c. each, 6 for $\$ 3.90$. " $F$ " is much better. Made of forged steel, patent spring. These are strong, substantial, and undoubtedly the best shears on the market. $\$ 1.00$ each, 6 for $\$ 5.75$

L a ls Following are the kinds mostly used by nurserymen. For 10,000 or more we allow a discount of 10 per cent. 1,000 3/2/2-in. and 1006 -in. in a package. Not less that a full package sold. $31 / 2-i n .$, notched, iron wired, painted $\ldots \ldots \ldots \ldots \ldots \ldots \ldots \ldots 1.00$ per 1,000 $31 / 2$-in., notched, copper wired, painted...................... $\$ 1.25$ per 1,000 6 -in., notched, painted, but not wired.................\$2.35 per 1,000

6 -in., notched, not painted or wired.................. $\$ 1.75$ per 1,000

Spades The Best Nursery Spade D Handle, Double Strap, Reinforced Nursery Spade. Weight $91 / 4$ makes of nursery spades, but find this to be the strongest, best and most durable on the market. It is strapped full length of handle, both back and front, and half way up it has double straps, strengthening it in what otherwise would be its weakest parts. When ordering state if straight or taper blade is wanted.

\section{Thomas Meehan \& Sons, Inc.}

